\title{
SN 1978K: An evolved supernova outside our Local Group detected at millimetre wavelengths
}

\author{
S. D. Ryder ${ }^{1}$, R. Kotak ${ }^{2}$, I. A. Smith ${ }^{3}$, S. J. Tingay ${ }^{4,5}$, E. C. Kool ${ }^{6,1}$, and J. Polshaw ${ }^{2}$ \\ 1 Australian Astronomical Observatory, PO Box 915, North Ryde, NSW 1670, Australia \\ e-mail: sdr@aao.gov.au \\ 2 Astrophysics Research Centre, School of Mathematics and Physics, Queen's University Belfast, BT7 1NN, UK \\ 3 Department of Physics and Astronomy, Rice University, 6100 South Main, MS-108, Houston, TX 77251-1892, USA \\ 4 Istituto di Radioastronomia, INAF, via P. Gobetti, 40129 Bologna, Italy \\ 5 International Centre for Radio Astronomy Research, Curtin University, Bentley, WA 6102, Australia \\ 6 Department of Physics and Astronomy, Macquarie University, Sydney, NSW 2109, Australia \\ Received 21 September 2016 / Accepted 6 October 2016
}

\begin{abstract}
Supernova $1978 \mathrm{~K}$ is one of the oldest-known examples of the class of Type IIn supernovae that show evidence for strong interaction between the blast wave and a dense, pre-existing circumstellar medium. Here we report detections of SN $1978 \mathrm{~K}$ at both $34 \mathrm{GHz}$ and $94 \mathrm{GHz}$, making it only the third extragalactic supernova (after SN 1987A and SN 1996cr) to be detected at late-times at these frequencies. We find SN 1978K to be $>400$ times more luminous than SN 1987A at millimetre wavelengths in spite of the roughly nine year difference in ages, highlighting the risk in adopting SN 1987A as a template for the evolution of core-collapse supernovae in general. Additionally, from new VLBI observations at $8.4 \mathrm{GHz}$, we measure a deconvolved diameter for SN $1978 \mathrm{~K}$ of $\sim 5 \mathrm{milli}$-arcsec, and a corresponding average expansion velocity of $<1500 \mathrm{~km} \mathrm{~s}^{-1}$. These observations provide independent evidence of an extremely dense circumstellar medium surrounding the progenitor star.
\end{abstract}

Key words. supernovae: general - supernovae: individual: SN 1978K - galaxies: individual: NGC 1313

\section{Introduction}

Following the first detection of a supernova at radio wavelengths close to fifty years ago (SN 1970G; Gottesman et al. 1972), it was soon widely realized that multi-frequency radio light curves can yield information about the configuration, density, and prevailing conditions of the circumstellar medium (e.g. Chevalier 1982b; Weiler et al. 1986). For cases where the radio light curves are well sampled, this information in turn can be translated into mass loss rates from the progenitor as a function of time, thereby revealing the likely evolutionary stages the star must have passed through prior to explosion (e.g. Sramek et al. 1984; Williams et al. 2002; Kotak \& Vink 2006). Additionally, carrying out very long baseline radio-interferometry (VLBI) on nearby ( $\lesssim$ few Mpc) objects can spatially resolve the expanding ejecta, uncovering underlying asymmetries, potentially due to a binary companion (e.g. Marcaide et al. 1994; Bartel \& Bietenholz 2003).

It is generally well established that the radio emission arises from a combination of synchrotron self-absorption and freefree absorption as the blast wave from the supernova encounters the surrounding medium ionized by the initial high energy flash from the explosion, but shaped by the progenitor system (Chevalier 1982a,b). At early epochs, the optical depth is large, suppressing the radio emission. As the shock moves outwards, the optical depth drops, and its frequency dependence results in the transition from optically thick to optically thin occurring first at higher frequencies. Post peak, the emission declines smoothly as a power-law, unless further dense material is encountered, perhaps from earlier mass loss episodes.
Although several hundred supernovae ( $\mathrm{SNe}$ ) have now been detected at radio wavelengths, the overwhelming majority of these are detections at early times. This can be attributed to a combination of intrinsic faintness and sensitivity of currently available instrumentation on the one hand, and the lack of circumstellar material (CSM) at large distances from the supernova that is dense enough to generate radio emission on the other.

Supernova 1978K (SN 1978K) in the late-type barred spiral galaxy NGC 1313 was only the second supernova to be detected and recognized as a supernova from its radio emission. and the first from its X-rays (Ryder et al. 1993). At peak, it would have been one of the brightest radio supernovae yet seen. The Cepheid-based distance to NGC 1313 is $4.61 \pm 0.21 \mathrm{Mpc}$ (Qing et al. 2015), although estimates based on the tip of the Red Giant Branch method (e.g. Jacobs et al. 2009) put it slightly closer. Regardless, SN 1978K is one of the closest supernovae undergoing ejecta-circumstellar interaction (Type IIn). It is one of a small number of $\mathrm{SNe}$ amenable to long term multiwavelength monitoring, and it remains bright at all wavelengths almost four decades after explosion.

In this Letter we report new high frequency, and high spatial resolution imaging of SN 1978K with the Australia Telescope Compact Array (ATCA) and Long Baseline Array (LBA).

\section{Observations and results}

\subsection{ATCA observations}

SN $1978 \mathrm{~K}$ was observed at $34 \mathrm{GHz}$ and at $94 \mathrm{GHz}$ with the ATCA in September 2014. The $34 \mathrm{GHz}$ observations were 
Table 1. ATCA observations of SN 1978K.

\begin{tabular}{lcc}
\hline \hline Parameter & $34 \mathrm{GHz}$ & $94 \mathrm{GHz}$ \\
\hline Date (UT) & 2014 Sep. 14 & 2014 Sep. 29 \\
Array configuration & $6 \mathrm{~B}$ & $\mathrm{H} 214$ \\
Max. baseline (m) & 5969 & 240 \\
No. of antennas & 6 & 5 \\
Total on-source time (h) & 7.6 & 5.6 \\
Synthesised beam (") & $0.44 \times 0.24$ & $1.9 \times 1.6$ \\
Image rms (mJy/beam) & 0.03 & 0.13 \\
Source flux density (mJy) & $2.9 \pm 0.2$ & $1.2 \pm 0.3$ \\
\hline
\end{tabular}

performed with the array in the $6 \mathrm{~B}$ configuration, and the $94 \mathrm{GHz}$ observations with the array in the more compact $\mathrm{H} 214$ configuration. The observations were carried out in two bands, each of $2 \mathrm{GHz}$ bandwidth, centred either on 33 and $35 \mathrm{GHz}$, or on 93 and $95 \mathrm{GHz}$, using the Compact Array Broad-band Backend (CABB: Wilson et al. 2011) with each $2 \mathrm{GHz}$ band consisting of $2048 \times 1 \mathrm{MHz}$ channels. The parameters of each observing session are listed in Table 1.

The observations consisted of 12 min scans on SN 1978K, interleaved with 2 min scans on the phase calibrator PKS B0302623 having flux densities of $\sim 0.8 \mathrm{Jy}$ at $34 \mathrm{GHz}$ and $\sim 0.6 \mathrm{Jy}$ at $94 \mathrm{GHz}$. The sources PKS B2155-152 and PKS B1921-293 served as the bandpass calibrators at $34 \mathrm{GHz}$ and at $94 \mathrm{GHz}$, respectively. Uranus was used as the primary flux density calibrator at both frequencies, with hourly pointing scans on the phase calibrator used to update the pointing model. At $94 \mathrm{GHz}$ "paddle" scans preceded each phase calibrator observation to monitor the system temperatures.

The data were processed using the MIRIAD ${ }^{1}$ package, and procedures outlined in Sect. 4.3 of the ATCA User Guide ${ }^{2}$. The $34 \mathrm{GHz}$ visibility data were corrected for atmospheric opacity, while the $94 \mathrm{GHz}$ visibility data had corrections applied to the antenna positions and system temperatures. After editing and calibration of the data, images at each frequency were made using a Briggs robust weighting factor of 0.5 as a best compromise between resolution and sensitivity, then cleaned down to three times the r.m.s. noise level. A clear detection of SN 1978K was obtained in each frequency, and fitting of a Gaussian point source yielded the flux densities shown in Table 1 where the uncertainty in each case is a combination of the fitting and absolute flux calibration errors. The resulting image of SN 1978K at $94 \mathrm{GHz}$ is shown in Fig. 1. Core-collapse supernovae have been detected previously at these frequencies (e.g. SN 2011dh: Horesh et al. 2013), but usually only within a few days or weeks after explosion.

In Fig. 2 we present the $1-100 \mathrm{GHz}$ spectral energy distribution (SED) of SN 1978K. The 1-10 GHz flux densities come from ongoing monitoring with the ATCA, the most recent epoch for which is 2013 June 7 UT. The expected flux densities at 1.4, 2.4, 4.8, and $8.6 \mathrm{GHz}$ for 2014 September have been derived using the model light curve fits in Smith et al. (2007). While a power-law index of $\alpha=-0.6$ (where flux density $S_{v} \propto v^{\alpha}$ ) provides a good fit to the data below $10 \mathrm{GHz}$, the power law steepens to $\alpha \sim-0.9$ between the two higher frequency data points. We note that even with the spectral steepening at high frequencies, extrapolation of this power law to the regime of the Atacama Large Millimetre/submillimetre Array (ALMA) Bands 4

\footnotetext{
1 http://www.atnf.csiro.au/computing/software/miriad/

2 http://www.narrabri.atnf.csiro.au/observing/users_ guide/html/atug.html
}

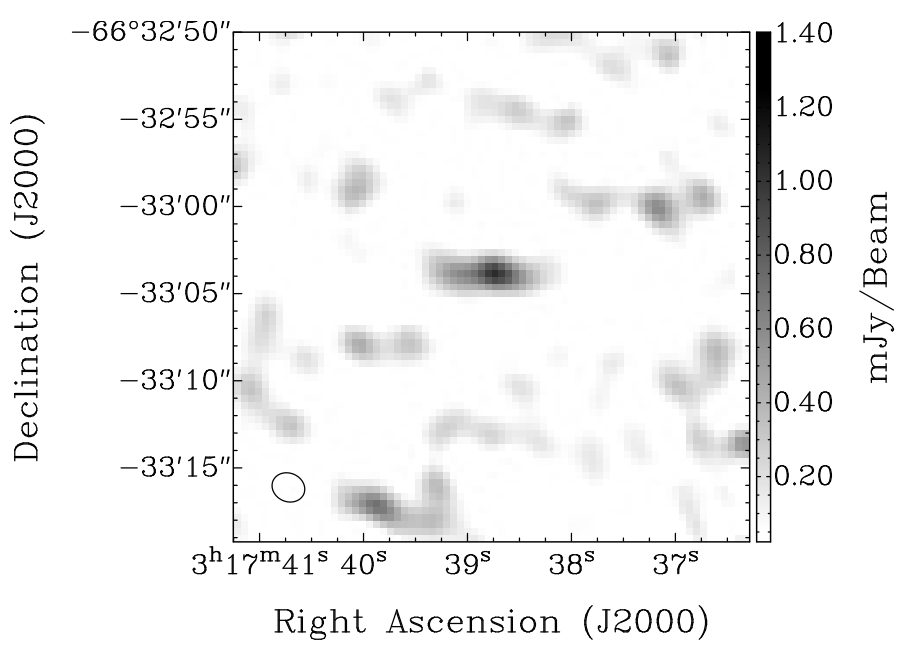

Fig. 1. Continuum image of SN $1978 \mathrm{~K}$ at $94 \mathrm{GHz}$ from the ATCA on 2014 Sep. 29 , showing a $10 \sigma$ detection at the $\mathrm{SN}$ location $\left(03^{\mathrm{h}} 17^{\mathrm{m}} 38.8^{\mathrm{s}}\right.$, $\left.-66^{\circ} 33^{\prime} 04^{\prime \prime} \mathrm{J} 2000\right)$. The synthesised beam is indicated in the lower-left corner and the apparent source elongation is an artifact of the underlying noise pattern.

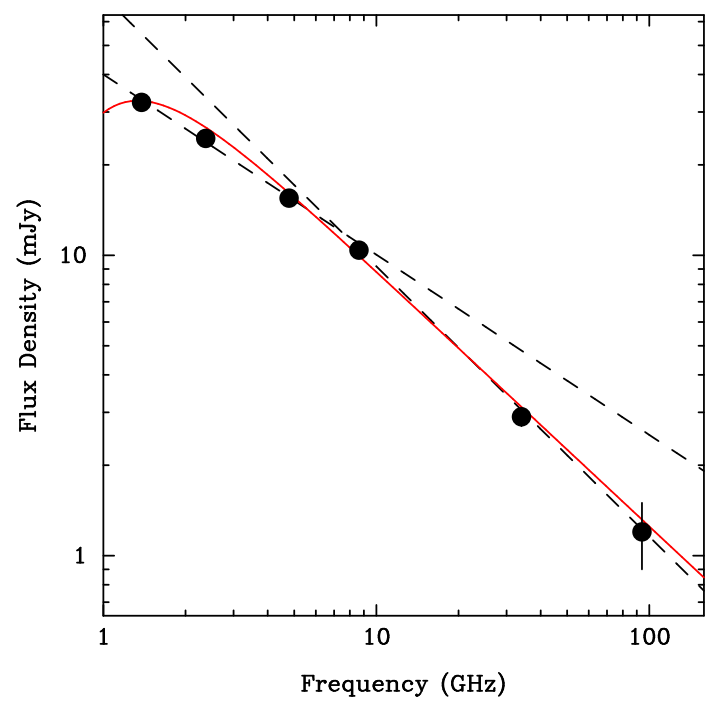

Fig. 2. Radio spectral energy distribution for SN 1978K in September 2014. The dashed lines mark a power law slope of -0.6 passing through the $v<10 \mathrm{GHz}$ data, and a steeper power law with slope of -0.9 passing through the $v>10 \mathrm{GHz}$ data. The red solid line shows an SED fit to the full dataset using the model of Weiler et al. (2002), which includes low frequency absorption due to an H II region along the line of sight.

(144 GHz) through $7(325 \mathrm{GHz})$ should make the continuum emission from SN $1978 \mathrm{~K}$ easily detectable with ALMA. The Type IIn SN 1996cr (Meunier et al. 2013) in the Circinus Galaxy lies at a distance comparable to SN 1978K; it was found using the ATCA to have flux densities of $23.9 \pm 6.0 \mathrm{mJy}$ at $88.6 \mathrm{GHz}$ at 3616 days after the explosion, and $40.3 \pm 6.0 \mathrm{mJy}$ at $34.5 \mathrm{GHz}$ at 4842 days. The flux densities measured recently for SN 1987A at $44 \mathrm{GHz}$ (Zanardo et al. 2013) and at $94 \mathrm{GHz}$ (Lakićević et al. 2012) would, if it were placed at the distance of NGC 1313 instead of $51.4 \mathrm{kpc}$ (Panagia 1999), correspond to $\sim 5 \mu \mathrm{Jy}$ and $\sim 3 \mu \mathrm{Jy}$ respectively, more than 2 orders of magnitude fainter than observed for SN 1978K.

\subsection{VLBI observations}

SN 1978 K was observed with the LBA on 2007 Nov. 13 and 2015 March 29 UT. These gave similar results and we 
discuss only the latter here because of the longer time since explosion. For this observation the array was comprised of 6 antennas: ATCA $5 \times 22 \mathrm{~m}$ phased array; Parkes $64 \mathrm{~m}$; Mopra $22 \mathrm{~m}$; Ceduna $30 \mathrm{~m}$; Hobart $26 \mathrm{~m}$; and a single $12 \mathrm{~m}$ antenna of the Australian Square Kilometre Array Pathfinder (ASKAP) in Western Australia, offering baselines in excess of $3000 \mathrm{~km}$. Although the observation block spanned $12 \mathrm{~h}$, the source was only above the elevation limit of some antennas for $\sim 10 \mathrm{~h}$. A bandwidth of $64 \mathrm{MHz}$ centred on $8.4 \mathrm{GHz}$ was used in dual polarisation mode, and data recorded to disk. The sources PKS B1921293 and PKS B0537-441 served as fringe-finders, while 3 min scans of SN 1978K were alternated with equivalent scans of the nearby source PKS B0355-669 $(\sim 0.5 \mathrm{Jy}$ at $8.4 \mathrm{GHz})$ to allow phase referencing.

All the data were correlated using the DiFX software correlator (Deller et al. 2011), and the correlated data were exported to $\mathrm{AIPS}^{3}$ for editing, applying nominal sensitivities for each antenna, and iterative fringe-fitting. Imaging using standard selfcalibration, robust $=0$ weighting, and clean techniques was carried out with the IMAGR task. The resultant image (Fig. 3) has a beam size of $\sim 3$ milli-arcsec and a dynamic range of $\sim 100: 1$. Deconvolution of the VLBI source in the image plane using the JMFIT task yields a deconvolved Gaussian size of 4.9 mas $\times$ 4.4 mas $( \pm 0.5$ mas in each axis $)$ at a position angle of $(20 \pm 40)^{\circ}$ compared to the beam position angle of $85^{\circ}$. The integrated flux density of $5.2 \pm 0.3 \mathrm{mJy}$ is just half the ATCA-measured value at $9 \mathrm{GHz}$ in Fig. 2, suggesting that some of the more-extended emission has been resolved out on account of the longer baselines and sparse $u v$-coverage. Fitting of the visibilities with a model in the $u v$-plane yielded similar results.

\section{Discussion}

\subsection{Radio SED}

While the $1-100 \mathrm{GHz}$ radio spectrum of SN 1987A is consistent with a single power law having $\alpha=-0.74$ (Zanardo et al. 2013), Fig. 2 shows this is clearly not the case for SN 1978K. A break such as this is not unusual in core collapse supernovae at early phases, where synchrotron self-absorption and/or free-free absorption results in a flattening of the spectral index at lower frequencies that are still optically-thick. However, after $36 \mathrm{yr}$ the expectation would be that SN $1978 \mathrm{~K}$ should be optically thin at all these frequencies.

Montes et al. (1997) noted a similar curvature in the 0.8-9 GHz spectrum of SN $1978 \mathrm{~K}$ at $\sim 5000$ days, which they attributed to an additional time-independent free-free absorption component along the line-of-sight, namely a foreground $\mathrm{H}$ II region. Using their Eq. (4) for the SED, but with model parameters from our fit to the longer time baseline data in Smith et al. (2007) we are able to reproduce the observed low frequency turnover as shown in Fig. 2 using a value for the thermal optical depth parameter $K_{4}$ that is $2.5 \times$ larger than that determined by Montes et al. (1997). This is nevertheless still consistent with the presence of an intervening H II region, or a circumstellar ejecta nebula as argued by Chu et al. (1999) on the basis of optical spectra. Observations at even lower frequencies would help to further constrain this fit, but the GaLactic and Extragalactic All-Sky MWA Survey (GLEAM; Wayth et al. 2015) at 70-230 MHz currently lacks the necessary resolution to cleanly separate SN 1978K from its host galaxy emission.

\footnotetext{
http://www. aips.nrao.edu/index.shtml
}

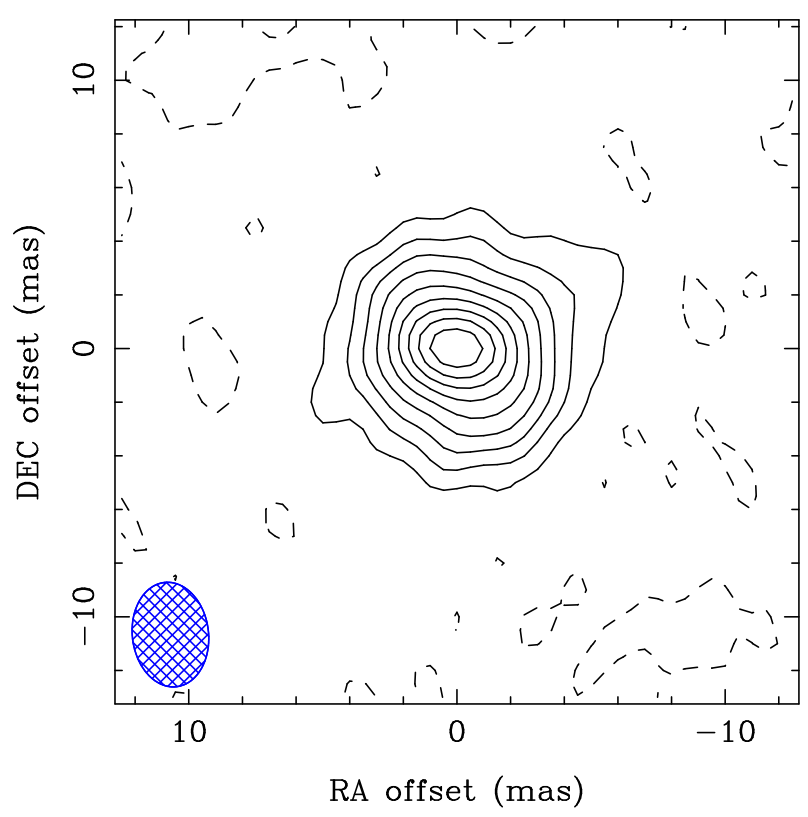

Fig. 3. VLBI image of SN $1978 \mathrm{~K}$ at a frequency of $8.4 \mathrm{GHz}$ on 2015 March 29. The map is centered at 03:17:38.62, -66:33:03.4 (J2000). The beam shown at lower-left by the hashed ellipse is 3.9 mas $\times$ 2.8 mas. Contours are shown at $-2 \%, 10 \%, 20 \%, 30 \%, \ldots, 90 \%$ of the peak brightness of $1.95 \mathrm{mJy} /$ beam.

\subsection{Expansion velocity}

Were SN 1978K undergoing free expansion at a mean velocity of $10000 \mathrm{~km} \mathrm{~s}^{-1}$, it should span nearly 40 mas and be easily resolvable by now. Our previous LBA observations at $1.4 \mathrm{GHz}$ and $2.3 \mathrm{GHz}$ of SN 1978K in 2003 (Smith et al. 2007) put an upper limit on the expanding remnant diameter of $\sim 10$ mas, or $0.22 \mathrm{pc}$ at the distance of NGC 1313. It appears that more than a decade later SN $1978 \mathrm{~K}$ is still barely resolved even at $8.4 \mathrm{GHz}$, with no apparent shell or ring-like structure yet visible as seen in SN 1987A (Zanardo et al. 2013) or SN 1993J (Marcaide et al. 2009a). We can, however, halve the upper limit on the diameter to $<5$ mas $(0.11 \mathrm{pc})$, and consequently the average expansion velocity for SN $1978 \mathrm{~K}$ since explosion is $<1500 \mathrm{~km} \mathrm{~s}^{-1}$. While much lower than the $8000-10000 \mathrm{~km} \mathrm{~s}^{-1}$ average expansion velocity inferred from VLBI measurements of objects like SN 1979C (Marcaide et al. 2009b) and SN 1986J (Bietenholz et al. 2010) some $25 \mathrm{yr}$ after their explosions, we note the recently measured range of current expansion velocities for SN 1987A of between 1600 and $6300 \mathrm{~km} \mathrm{~s}^{-1}$ depending on azimuth (Zanardo et al. 2013). This confirms that the radio remnant of SN 1987A has also undergone significant deceleration since the initial explosion at $\sim 35000 \mathrm{~km} \mathrm{~s}^{-1}$ (Gaensler et al. 1997).

We can make use of this new VLBI constraint on the current diameter of the remnant of SN 1978K, together with information from the radio light curve fits, to infer how the ejecta has decelerated over time. The blast wave radius is assumed to grow with time as $R \sim t^{m}$, where $m=1$ for no deceleration. In the Weiler et al. (2002) parameterisation of the Chevalier (1982a) model $m=-(\alpha-\beta-3) / 3$, where $\alpha$ is the radio spectral index, and $\beta$ is the rate of decline in flux density during the post-peak, optically-thin phase. The SED fit shown in Fig. 2 has $\alpha=-0.85$ and $\beta=-1.51$, so $m=-0.78$ which is at the extreme end for decelerations measured this way (Weiler et al. 2002).

Figure 4 shows a model of the evolution in diameter, current expansion velocity, and past average expansion velocity of 


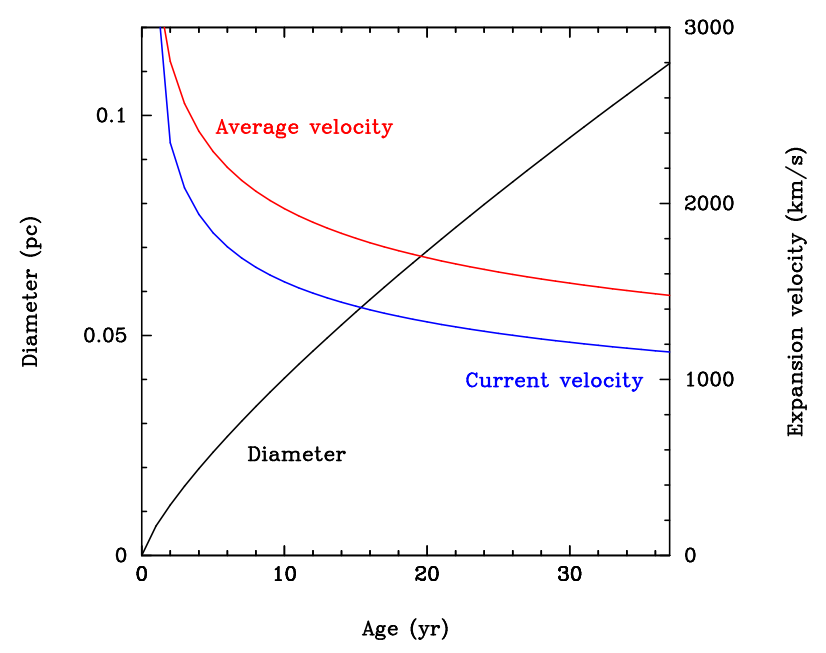

Fig. 4. Modelled evolution of the remnant diameter of SN 1978K (black line), together with the current (blue line) and past average (red line) expansion velocities, for $R \sim t^{0.78}$

SN $1978 \mathrm{~K}$ for $R \sim t^{0.78}$ such that the current diameter is no more than $0.1 \mathrm{pc}$. Recent optical spectroscopy of SN $1978 \mathrm{~K}$ by Kuncarayakti et al. (2016) shows remarkable similarities with SN 1987A, and line-widths indicating a current expansion velocity $\sim 500-600 \mathrm{~km} \mathrm{~s}^{-1}$, within a factor of two of that predicted by this model. Whereas the radio emission at such late times is thought to trace the forward shock, the optical emission lines are associated with the slower-moving reverse shock. Kuncarayakti et al. (2016) inferred an extreme mass-loss rate $\left(0.01 M_{\odot} \mathrm{yr}^{-1}\right)$ based on the $\mathrm{H} \alpha$ luminosity, leading to a massive circumstellar medium $\left(\sim 1 M_{\odot}\right)$. Although there are several caveats that need to be considered when converting $\mathrm{H} \alpha$ luminosities to mass loss rates, the values above could simultaneously account for the significant radio luminosity as well as the dramatic deceleration in the expansion. Nevertheless we note that while Type IIn supernovae are among the most luminous at radio wavelengths, SN 1978K is in fact not unusually luminous for its type (e.g. Romero-Cañizales et al. 2014).

\section{Conclusions}

New radio observations of the Type IIn SN 1978K at high frequencies and at high spatial resolution have led to some intriguing results:

1. SN $1978 \mathrm{~K}$ turns out to be $>400 \times$ brighter at frequencies above $30 \mathrm{GHz}$ than SN 1987A, the only other extragalactic supernova to have been detected at these frequencies more than 2 decades after explosion.

2. A single power-law does not fit the $1-100 \mathrm{GHz}$ SED of SN 1978K. This may be because the intrinsic emission has a broken power law, or due to the presence of a nebular absorber along the line of sight as previously suspected from low-frequency observations. Future metre-wave and sub-millimetre observations should be able to discriminate between these two scenarios.
3. The remnant of SN $1978 \mathrm{~K}$ is an order of magnitude smaller than expected for the case of free expansion, and instead appears to be decelerating as $R \sim t^{0.78}$. The past average expansion rate is below $1500 \mathrm{~km} \mathrm{~s}^{-1}$, and the current expansion rate modelled to be $20 \%$ lower still.

These findings lend support to the arguments from contemporary X-ray and optical analyses that the progenitor star of SN $1978 \mathrm{~K}$ underwent extreme mass-loss in its final centuries before explosion. This material is responsible for drastically decelerating the expansion of the remnant in much the same way as observed in SN 1987A, which shows other similarities with the older SN 1978K despite not being classed as a Type IIn event.

Acknowledgements. S.R. and R.K. acknowledge support from the Royal Society International Exchange scheme (IE140343). S.R. was a Visiting Research Fellow at Queen's University Belfast where much of this paper was written. R.K. also acknowledges support from STFC via ST/L000709/1. We are grateful to Cormac Reynolds for invaluable assistance with reducing the LBA data. We thank F. Bauer for bringing to our attention the millimetre observations of SN 1996cr. The Australia Telescope Compact Array and Long Baseline Array are part of the Australia Telescope National Facility which is funded by the Commonwealth of Australia for operation as a National Facility managed by CSIRO. The data reported here were obtained under Programs C184 and V157 (P.I. S. Ryder).

\section{References}

Bartel, N., \& Bietenholz, M. F. 2003, ApJ, 591, 301

Bietenholz, M. F., Bartel, N., \& Rupen, M. P. 2010, ApJ, 712, 1057

Chevalier, R. A. 1982a, ApJ, 258, 790

Chevalier, R. A. 1982b, ApJ, 259, 302

Chu, Y.-H., Caulet, A., Montes, M. J., et al. 1999, ApJ, 512, L51

Deller, A. T., Brisken, W. F., Phillips, C. J., et al. 2011, PASP, 123, 275

Gaensler, B. M., Manchester, R. N., Staveley-Smith, L., et al. 1997, ApJ, 479, 845

Gottesman, S. T., Broderick, J. J., Brown, R. L., Balick, B., \& Palmer, P. 1972, ApJ, 174, 383

Horesh, A., Stockdale, C., Fox, D. B., et al. 2013, MNRAS, 436, 1258

Jacobs, B. A., Rizzi, L., Tully, R. B., et al. 2009, AJ, 138, 332

Kotak, R., \& Vink, J. S. 2006, A\&A, 460, L5

Kuncarayakti, H., Maeda, K., Anderson, J. P., et al. 2016, MNRAS, 458, 2063

Lakićević, M., Zanardo, G., van Loon, J. T., et al. 2012, A\&A, 541, L2

Marcaide, J. M., Alberdi, A., Elosegui, P., et al. 1994, ApJ, 424, L25

Marcaide, J. M., Martí-Vidal, I., Alberdi, A., et al. 2009a, A\&A, 505, 927

Marcaide, J. M., Martí-Vidal, I., Perez-Torres, M. A., et al. 2009b, A\&A, 503, 869

Meunier, C., Bauer, F. E., Dwarkadas, V. V., et al. 2013, MNRAS, 431, 2453

Montes, M. J., Weiler, K. W., \& Panagia, N. 1997, ApJ, 488, 792

Panagia, N. 1999, in New Views of the Magellanic Clouds, eds. Y.-H. Chu, N. Suntzeff, J. Hesser, \& D. Bohlender, IAU Symp., 190, 549

Qing, G., Wang, W., Liu, J.-F., \& Yoachim, P. 2015, ApJ, 799, 19

Romero-Cañizales, C., Herrero-Illana, R., Pérez-Torres, M. A., et al. 2014, MNRAS, 440, 1067

Ryder, S., Staveley-Smith, L., Dopita, M., et al. 1993, ApJ, 416, 167

Smith, I. A., Ryder, S. D., Böttcher, M., et al. 2007, ApJ, 669, 1130

Sramek, R. A., Panagia, N., \& Weiler, K. W. 1984, ApJ, 285, L59

Wayth, R. B., Lenc, E., Bell, M. E., et al. 2015, PASA, 32, e025

Weiler, K. W., Sramek, R. A., \& Panagia, N. 1986, Science, 231, 1251

Weiler, K. W., Panagia, N., Montes, M. J., \& Sramek, R. A. 2002, ARA\&A, 40, 387

Williams, C. L., Panagia, N., Van Dyk, S. D., et al. 2002, ApJ, 581, 396

Wilson, W. E., Ferris, R. H., Axtens, P., et al. 2011, MNRAS, 416, 832

Zanardo, G., Staveley-Smith, L., Ng, C.-Y., et al. 2013, ApJ, 767, 98 